5. Yang HP, Cook LS, Weiderpass E, et al. Infertility and incident endometrial cancer risk: a pooled analysis from the Epidemiology of Endometrial Cancer Consortium (E2C2). Br J Cancer. 2015;112(5):925-933.

\section{Mechanical Thrombectomy and Functional Outcomes After Stroke}

To the Editor The 2015 American Heart Association/American Stroke Association focused update for the early management of acute ischemic stroke recommended endovascular therapy as an adjunctive therapy to intravenous thrombolysis. ${ }^{1}$ Dr Badhiwala and colleagues ${ }^{2}$ performed a meta-analysis regarding endovascular thrombectomy for acute ischemic stroke and found that endovascular thrombectomy was associated with improved functional outcomes (odds ratio [OR], 1.56 [95\% CI, 1.14-2.13]; $P=.005)$ and no significant difference in allcause mortality (OR, 0.87 [95\% CI, 0.68-1.12]; $P=.27$ ) at 90 days compared with standard medical care. The authors included the SYNTHESIS (Intra-arterial vs Systemic Thrombolysis for Acute Ischemic Stroke) expansion trial, ${ }^{3}$ which randomized patients with acute ischemic stroke to receive endovascular therapy alone (without intravenous tissue plasminogen activator [tPA] vs tPA. We argue that the SYNTHESIS study ${ }^{3}$ should have been excluded from the analysis because it is fundamentally different from the other included studies. Including SYNTHESIS in the Badhiwala et al analysis could have underestimated the effect of interventional treatment on important outcomes.

A similar meta-analysis evaluating the efficacy of endovascular thrombectomy in acute ischemic stroke excluded the SYNTHESIS expansion trial and also included 2 recently presented studies (ie, THERAPY [Randomized, Concurrent Controlled Trial to Assess the Penumbra System's Safety and Effectiveness in the Treatment of Acute Stroke] and THRACE [Trial and Cost Effectiveness Evaluation of Intraarterial Thrombectomy in Acute Ischemic Stroke]). ${ }^{4}$ This analysis demonstrated improvement in functional outcomes (risk ratio [RR], 1.45 [95\% CI, 1.22-1.72]; $P<.001$ ) with endovascular thrombectomy. Thus, thrombectomy for acute stroke appears to be beneficial. However, accurate treatment summary estimates depend on correct selection of trials for analysis.

\section{Islam Y. Elgendy, MD \\ Deepak L. Bhatt, MD, MPH \\ Anthony A. Bavry, MD, MPH}

Author Affiliations: Department of Medicine, University of Florida, Gainesville (Elgendy, Bavry); Brigham and Women's Hospital Heart and Vascular Center, Harvard Medical School, Boston, Massachusetts (Bhatt).

Corresponding Author: Anthony A. Bavry, MD, MPH, North Florida/South Georgia Veterans Health System (Malcom Randall VA Medical Center), Medical Service, Cardiology Section (111D), 1601 SW Archer Rd, Gainesville, FL 32608 (anthony.bavry@va.gov).

Conflict of Interest Disclosures: The authors have completed and submitted the ICMJE Form for Disclosure of Potential Conflicts of Interest. Dr Bhatt reports serving on the advisory boards for Cardax, Elsevier Practice Update Cardiology, Medscape Cardiology, and Regado Biosciences; serving on the board of directors for Boston VA Research Institute and the Society of Cardiovascular Patient Care; being the chair of the American Heart Association Quality Oversight Committee; serving on the data monitoring committees of the Duke
Clinical Research Institute, Harvard Clinical Research Institute, Mayo Clinic, and Population Health Research Institute; receiving honoraria from the American College of Cardiology, Belvoir Publications, Duke Clinical Research Institute, Harvard Clinical Research Institute, HMP Communications, Journal of the American College of Cardiology, Population Health Research Institute, Slack Publications, Society of Cardiovascular Patient Care, WebMD; serving as the deputy editor of Clinical Cardiology; receiving research funding from Amarin AstraZeneca, Bristol-Myers Squibb, Eisai, Ethicon, Forest Laboratories, Ischemix, Medtronic, Pfizer, Roche, sanofi-aventis, and The Medicines Company; receiving royalties from Elsevier; being a site coinvestigator for Biotronik and St Jude Medical; being a trustee of the American College of Cardiology; and participating in unfunded research for FlowCo, PLx Pharma, and Takeda. Dr Bavry reports receiving honorarium from the American College of Cardiology. No other disclosures were reported.

1. Powers WJ, Derdeyn CP, Biller J, et al; American Heart Association Stroke Council. 2015 American Heart Association/American Stroke Association Focused Update of the 2013 Guidelines for the Early Management of Patients With Acute Ischemic Stroke Regarding Endovascular Treatment: A Guideline for Healthcare Professionals From the American Heart Association/American Stroke Association. Stroke. 2015;46(10):3020-3035.

2. Badhiwala JH, Nassiri F, Alhazzani W, et al. Endovascular thrombectomy for acute ischemic stroke: a meta-analysis. JAMA. 2015;314(17):1832-1843.

3. Ciccone A, Valvassori L, Nichelatti M, et al; SYNTHESIS Expansion Investigators. Endovascular treatment for acute ischemic stroke. N Engl J Med. 2013;368(10):904-913.

4. Elgendy IY, Kumbhani DJ, Mahmoud A, Bhatt DL, Bavry AA. Mechanical thrombectomy for acute ischemic stroke: a meta-analysis of randomized trials. J Am Coll Cardiol. 2015;66(22):2498-2505.

To the Editor Dr Badhiwala and colleagues ${ }^{1}$ followed the Cochrane Collaboration standards to conduct a systematic review on the use of mechanical thrombectomy in acute ischemic stroke. Reviewing 8 randomized clinical trials, the authors concluded that, in selected patients, endovascular thrombectomy was associated with improved functional outcomes over standard medical care, without differences in adverse events.

All included studies used a prospective randomized open blinded end point (PROBE) design ${ }^{2}$ rather than an investigator-participant-evaluator blind design, and 5 studies were stopped early, 4 for benefit. The Cochrane Collaboration tool for assessing risk of bias and the Grades of Recommendation, Assessment, Development and Evaluation (GRADE) approach, ${ }^{3-5}$ both used by the authors, state that a "low risk of bias" should not be endorsed when selection, performance, or other forms of bias are present. The PROBE design assumes reliable randomization to allocate participants to treatment groups but does not include blinding of patients or study personnel. ${ }^{2}$ More importantly, evidence suggests that trials stopped early for benefit often overestimate treatment effects. ${ }^{4}$

We believe that studies without allocation concealment or blinding of participants and personnel and with other sources of bias cannot accurately be rated as high quality, as Badhiwala and colleagues did. Selection, performance and other biases would warrant a downgrading of the risk of bias item. If the quality rating fell by 1 level for each downgraded factor, ${ }^{5}$ this could change the quality of evidence from a moderate-to-high level to a moderate level, implying that further research is still needed. This reduction may have an important effect on the level of confidence in the estimate of effect ${ }^{4,5}$ and consequently in the way different stakehold- 
ers (clinicians, patients, and policy makers) perceive the health effect of a given therapeutic intervention.

\section{Filipe Brogueira Rodrigues, MD \\ Peter Langhorne, $\mathrm{PhD}$ \\ João Costa, MD, PhD}

\begin{abstract}
Author Affiliations: Clinical Pharmacology Unit, Instituto de Medicina Molecular, Lisbon, Portugal (Rodrigues); Institute of Cardiovascular and Medical Sciences, University of Glasgow, Glasgow, United Kingdom (Langhorne); Center for Evidence Based Medicine, University of Lisbon, Lisbon, Portugal (Costa)
\end{abstract}

Corresponding Author: João Costa, MD, PhD, Cochrane Movement Disorders Group, Laboratory of Clinical Pharmacology and Therapeutics, Faculty of Medicine, University of Lisbon, Av Professor Egas Moniz, 1649-028, Lisbon, Portugal (jncosta@fm.ul.pt).

Conflict of Interest Disclosures: The authors have completed and submitted the ICMJE Form for Disclosure of Potential Conflicts of Interest. Drs Rodrigues and Costa reported being involved in meta-research on the subject of neurovascular neurology. Dr Rodrigues also reported being an external editor of the Cochrane Movement Disorders Group. Dr Langhorne reported being the coordinating editor of the Cochrane Stroke Group. Dr Costa reported being the coordinating editor of the Cochrane Movement Disorders Group and coordinator director of Cochrane Portugal.

Disclaimer: This letter is solely the responsibility of the authors and does not necessarily represent the views of Cochrane or any other entity with which the authors are associated.

1. Badhiwala JH, Nassiri F, Alhazzani W, et al. Endovascular thrombectomy for acute ischemic stroke: a meta-analysis. JAMA. 2015;314(17):1832-1843.

2. Hansson L, Hedner T, Dahlöf B. Prospective randomized open blinded end-point (PROBE) study: a novel design for intervention trials. Blood Press. 1992;1(2):113-119.

3. Ioannidis JP, Fanelli D, Dunne DD, Goodman SN. Meta-research: evaluation and improvement of research methods and practices. PLOS Biol. 2015:13(10): e1002264.

4. Pollock A, Farmer SE, Brady MC, et al. An algorithm was developed to assign GRADE levels of evidence to comparisons within systematic reviews. J Clin Epidemiol. 2016;70(10):106-110.

5. Higgins JPT, Green S, eds. Cochrane Handbook for Systematic Reviews of Interventions. http://handbook.cochrane.org. Accessed September 1, 2015

In Reply Dr Elgendy and colleagues suggest that the SYNTHESIS $^{1}$ trial should have been excluded from our metaanalysis because the endovascular therapy group did not receive intravenous tPA. At the time SYNTHESIS ${ }^{1}$ and the Interventional Management of Stroke III (IMS III) ${ }^{2}$ were designed, there was limited data on combining intravenous tPA with endovascular thrombectomy. ${ }^{3}$ The rationale for combination therapy was the yet unproven assumption that endovascular thrombectomy and intravenous tPA could work in synergy; however, there was also concern about a possible higher risk of hemorrhagic transformation, especially at full doses of tPA. Reflecting these opposing hypotheses, the SYNTHESIS $^{1}$ trial aimed to evaluate direct endovascular intervention, whereas IMS III $^{2}$ sought to test the safety and efficacy of endovascular therapy after intravenous thrombolysis. With the state of the available literature, we thought it would be most informative to perform an overall pooled analysis of endovascular thrombectomy (with or without intravenous tPA) vs standard medical care, and subsequent analyses stratified by concurrent use of intravenous tPA with mechanical thrombectomy. We found more favorable func- tional outcomes when thrombectomy was combined with intravenous tPA than when performed in isolation. This has important implications on a systems level, as it lends support to "drip-and-ship" (tPA intravenous drip and shipping patients to the angiogram catheter suite for endovascular thrombectomy) paradigms of care. Moreover, Elgendy and colleagues allude to the THERAPY and THRACE trials. Both studies have yet to be published, and only intermediary results from THRACE are available. The inclusion of incomplete data from these trials, yet selective exclusion of SYNTHESIS, ${ }^{1}$ could bias their meta-analysis. ${ }^{4}$

Dr Rodrigues and colleagues suggest that the rating of the quality of evidence in our meta-analysis should be changed from moderate to high to moderate because all trials used a PROBE design and because 4 trials were stopped early for benefit. However, it would not have been feasible to blind participants and clinicians, given that the intervention requires an operative procedure whereas the control therapy does not. The use of a sham procedure would pose an ethical conundrum. Furthermore, the lack of blinding is unlikely to have biased treatment effect estimates, considering that outcome assessors were blinded, there was no evidence to suggest cointervention or differential behavior by clinicians, and objective outcomes were used (eg, modified Rankin Scale [mRS] score, revascularization, mortality, intracerebral hemorrhage), which are less susceptible to bias by the placebo effect. ${ }^{5}$ Similarly, although trials stopped early for benefit may overestimate the treatment effect, this is mostly an issue when event numbers are small. ${ }^{6}$ The primary outcome in the trials stopped early, except 1 , was the mRS score. This outcome was examined using the proportional odds ratio (shift analysis), which takes into account the sum distribution of all mRS scores. This outcome, being derived from large event numbers, is less prone to overestimation. The factors Rodrigues and colleagues point out can introduce bias into any trial, but they do not in themselves indicate or ensure bias. A more careful look reveals that these characteristics are unlikely to have contributed significant bias to the results in this specific setting. We maintain that the quality of the evidence is moderate to high.

\section{Jetan H. Badhiwala, MD \\ Farshad Nassiri, MD}

Saleh A. Almenawer, MD

Author Affiliations: Division of Neurosurgery, University of Toronto, Toronto, Ontario, Canada (Badhiwala, Nassiri); Division of Neurosurgery, McMaster University, Hamilton, Ontario, Canada (Almenawer).

Corresponding Author: Saleh A. Almenawer, MD, Division of Neurosurgery, Department of Clinical Epidemiology and Biostatistics, McMaster University, 1280 Main St W, Hamilton, Ontario, Canada L8S 4L8 (dr_menawer@hotmail.com).

Conflict of Interest Disclosures: The authors have completed and submitted the ICMJE Form for Disclosure of Potential Conflicts of Interest and none were reported.

1. Ciccone A, Valvassori L, Nichelatti M, et al; SYNTHESIS Expansion Investigators. Endovascular treatment for acute ischemic stroke. N Engl J Med. 2013;368(10):904-913

2. Broderick JP, Palesch YY, Demchuk AM, et al; Interventional Management of Stroke (IMS) III Investigators. Endovascular therapy after intravenous t-PA versus t-PA alone for stroke. N Engl J Med. 2013;368(10):893-903. 
3. Bhatia R, Shobha N, Menon BK, et al. Combined full-dose IV and endovascular thrombolysis in acute ischemic stroke. Int J Stroke. 2014;9(8): 974-979.

4. Elgendy IY, Kumbhani DJ, Mahmoud A, Bhatt DL, Bavry AA. Mechanical thrombectomy for acute ischemic stroke: a meta-analysis of randomized trials. J Am Coll Cardiol. 2015;66(22):2498-2505.

5. Wood L, Egger M, Gluud LL, et al. Empirical evidence of bias in treatment effect estimates in controlled trials with different interventions and outcomes: meta-epidemiological study. BMJ. 2008;336(7644):601-605.

6. Montori VM, Devereaux PJ, Adhikari NK, et al. Randomized trials stopped early for benefit: a systematic review. JAMA. 2005;294(17):2203-2209.

\section{Conflict or Confluence of Interest?}

To the Editor A recent Viewpoint ${ }^{1}$ suggested that "the term conflict of interest is pejorative" and proposed using "confluence of interest" instead, along with visually mapping the complex system of biases within and around research. The material is thought-provoking but problematic.

To us it is far from clear that conflict of interest is a depreciative term. Although there are biasing factors other than conflicts of interest, ${ }^{2}$ the phrase captures the difficult nature of the topic and is not pejorative when correctly used to speak of perceived or potential-not actual-conflicts. Furthermore, confluence of interest is itself a problematic term. It implies that interests flow together, whereas reality suggests that some interests can counteract or outweigh others. The authors seem to believe that "implying an alignment of primary and secondary interests" for which biasing factors are not seen as problematic would be preferable to admitting that potential conflicts exist, but they did not offer any arguments for this position. The objective might be to minimize biasing factors, but renaming the term used to refer to biases while the objective is far from being met could undermine all the work done toward encouraging researchers to declare potential conflicts. ${ }^{3,4}$ To take a different example, if there was a problem with the terminology "fabrication of results," would it be renamed "full and transparent reporting" simply because the latter is the ultimate aim in combating the former? This would not seem appropriate, and the same applies to conflict of interest.

Moreover, although details of the terrain-mapping approach and its usefulness remain unclear, it is surprising that the authors think placing a 3-dimensional terrain mapping of fame and fortune resembling a heat map on consent forms would be a "simple and accessible" approach. Consent forms are already overlong and overcomplicated without giving potential study participants visual representations of how fame and fortune may bias investigators, institutions, funders, and journals in the complex interplay ${ }^{5}$ of research.

Ultimately, renaming conflicts of interest "confluence of interest" would do more harm than good and the proposed terrain mapping may complicate the issue beyond reasonable limits.

David M. Shaw, PhD

Peter Morfeld, PhD

Thomas C. Erren, MD

Author Affiliations: Institute for Biomedical Ethics, University of Basel, Basel, Switzerland (Shaw); Institute for Occupational Epidemiology and Risk Assessment, Evonik Industries AG, Essen, Germany (Morfeld); Institute and
Policlinic for Occupational Medicine, Environmental Medicine and Prevention Research, University of Cologne, Cologne, Germany (Erren).

Corresponding Author: David M. Shaw, PhD, Institute for Biomedical Ethics, University of Basel, Bernoullistrasse 28, 4056 Basel, Switzerland (david.shaw@unibas.ch).

Conflict of Interest Disclosures: The authors have completed and submitted the ICMJE Form for Disclosure of Potential Conflicts of Interest and none were reported.

1. Cappola AR, FitzGerald GA. Confluence, not conflict of interest: name change necessary. JAMA. 2015;314(17):1791-1792.

2. Shaw DM. Beyond conflicts of interest: disclosing medical biases [A Piece of My Mind]. JAMA. 2014;312(7):697-698.

3. Shaw DM, Erren TC. Ten simple rules for protecting research integrity. PLoS Comput Biol. 2015;11(10):e1004388.

4. Erren TC. Competing interests: judged in perpetuity. Nature. 2012;488(7413): 590.

5. Erren TC, Shaw DM, Morfeld P. Analyzing the publish-or-perish paradigm with game theory: the prisoner's dilemma and a possible escape. [published online September 12, 2015]. Sci Eng Ethics. doi:10.1007/s11948-015-9701-x.

In Reply Dr Shaw and colleagues think that the term "conflict of interest" is not pejorative because the implication is that this infers the potential for conflict rather than its reality. We do not think so. A conflict is a clash, a quarrel, a squabble, or a disagreement, often of a protracted nature, not just the prospect of its occurrence. Journals require increasingly "full and transparent reporting," for example, by provision of metadata, but do not request authors to address a priori their "fabrication of results" unless they detect evidence that this has occurred.

We agree that the present consent forms "are already overlong and overcomplicated," yet they focus on fiscal forms of bias to which the investigator is potentially liable and do not address the diversity of relevant stakeholders and the lure of fame, perhaps even more distracting to many academic investigators than money alone. There has been considerable progress in the visualization of complex data in the genomic era. This approach can readily be harvested to display for patients, scientists, and regulators potential sources of bias that are pertinent to a body of work. It seems to us that some effort along these lines is long overdue; it would be an improvement on the current copious, wordy, and often impenetrable consent forms-a picture, after all, is worth a thousand words.

Everyone has biases. Rather than present these pejoratively, as a clash of values that undermines validity, it seems more constructive to mine the complexity of these biases, present them in an accessible fashion, and seek to determine whether they are confluent with the interests of patients, scientists, and regulators who might base their decisions on the results of a given piece of work.

Anne R. Cappola, MD, ScM

Garret A. FitzGerald, MD, FRS

Author Affiliations: Institute for Translational Medicine and Therapeutics, University of Pennsylvania Perelman School of Medicine, Philadelphia (Cappola, FitzGerald); Associate Editor, JAMA (Cappola).

Corresponding Author: Anne R. Cappola, MD, ScM, Perelman School of Medicine at the University of Pennsylvania, Translational Research Center, 12th Floor, 3400 Civic Center Blvd, Bldg 421, Philadelphia, PA 19104-5160 (acappola @mail.med.upenn.edu). 